\title{
The molecular basis of UDP-galactose-4-epimerase (GALE) deficiency galactosemia in Korean patients
}

Hyung-Doo Park, $M D^{1}$, Kyung Un Park, MD, PhD ${ }^{1,2}$, Jin Q Kim, MD, PhD ${ }^{1}$, Choong Ho Shin, MD, PhD ${ }^{3}$, Sei Won Yang MD, $\mathrm{PhD}^{3}$, Dong Hwan Lee $M D, \mathrm{PhD}^{4}$, Young-Han Song PhD ${ }^{5}$, and Junghan Song MD, PhD ${ }^{1,2}$

\begin{abstract}
Purpose: UDP-galactose-4-epimerase (GALE) deficiency galactosemia is an autosomal recessive disorder and the prevalence of the disease varies among ethnic groups. We aimed to investigate molecular characteristics of the Korean patients with attenuated GALE activity and elevated galactose-1-phosphate levels in blood. Methods: In order to characterize the molecular defects underlying GALE deficiency, the GALE gene of 7 patients showing severe activity decreases was sequenced. PCR-RFLP was performed to confirm the presence of the mutations identified by sequencing. Results: Nine mutations were identified: 8 missense mutations (p.A25V, p.R40C, p.D69E, p.E165K, p.R169W, p.R239W, p.G302D, and p.R335H) and one nonsense mutation (p.W336X). Except for p.R335H, all of these mutations are novel. Six patients were compound heterozygotes (p.D69E/p.G302D, p.R40C/p.R169W, p.D69E/p.E165K, p.R239W/p.R335H, p.A25V/p.R169W, and p.G302D/p.R335H) and the remaining patient had only one mutation (p.W336X/not detected). Thirty patients with moderately reduced GALE activity were also tested by PCR-RFLP for the presence of the above mutation, and mutations were detected in 17 of these 30 patients. The frequency of p.G302D (9/30), p.R239W (6/30) and p.R169W (5/30) in our Korean patients with GALE deficiency galactosemia was relatively high. Conclusions: We detected 9 mutations of the GALE gene in Korean galactosemia patients, and confirmed allelic heterogeneity in this disease. Genet Med 2005:7(9): 646-649.
\end{abstract}

Key Words: galactosemia, UDP-galactose-4-epimerase (GALE), mutation, Korean

\section{INTRODUCTION}

UDP-galactose-4-epimerase (GALE: EC 5.1.3.2) deficiency galactosemia (MIM\# 230350) is an autosomal recessive disorder and is diagnosed in newborn screening by an increase in galactose or galactose-1-phosphate levels and a decrease in GALE enzyme activity. The impairment of GALE in humans results in one of two clinically distinct forms of epimerase deficiency galactosemia : one benign or peripheral, ${ }^{1-3}$ the other severe or generalized. ${ }^{4-8}$ In its benign or peripheral form, ${ }^{1}$ epimerase deficiency is limited to red blood cells and uncultured white blood cells, and epimerase activities in liver, cultured skin fibroblasts, and activated lymphocytes are normal. This benign form of GALE deficiency appears to be relatively common among Japanese and black people compared to

\footnotetext{
From the ${ }^{1}$ Department of Laboratory Medicine, Seoul National University College of Medicine, Seoul, Korea; ${ }^{2}$ Department of Laboratory Medicine, Seoul National University Bundang Hospital, Gyeonggi-do, Korea; ${ }^{3}$ Department of Pediatrics, Seoul National University College of Medicine, Seoul, Korea; ${ }^{4}$ Department of Pediatrics, School of Medicine, Soonchunhyang University, Seoul, Korea; ${ }^{5}$ Ilsong Institute of Life Science, Hallym University, Anyang, Korea Dr. Junghan Song, Seoul National University Bundang, Hospital, 300 Gumi-dong, Bundanggu, Seongnam-si, Gyeonggi-do 463-707, Korea.

Received: July 5, 2005
}

Accepted: September 9, 2005

DOI: 10.1097/01.gim.0000194023.27802.2d nonblacks. ${ }^{2,9}$ In the generalized form, two patients, one Pakistani and the other Asian Muslim, have been described as presenting clinical symptoms in the newborn period similar to classical galactosemia including vomiting, jaundice, and hepatomegaly. ${ }^{4,5}$ Epimerase is deficient in all tissue types. A galactose restriction diet helpfully reduces acute symptoms, but long-term complications are not preventable. Recently, a unique patient with an intermediate GALE-deficiency phenotype has been reported showing impaired neurological function but none of the symptoms of acute toxicity. ${ }^{9}$

Since the human GALE gene was first cloned, ${ }^{10}$ a total of nine missense mutations have been identified in patients with GALE deficiency, including p.G90E, p.D103G, p.K257R, p.L313M, p.G319E, ${ }^{3}$ p.V94M, ${ }^{7}$ p.N34S, p.L183P, ${ }^{11}$ and p.R335H. ${ }^{12}$

Biochemical studies show three of these mutations (p.G90E, p.V94M, and p.L183P) produce proteins with defective enzyme activity. Even though a p.V94M mutation was reported to be associated with the generalized form of epimerase deficiency, ${ }^{7,8,13}$ the genotype/phenotype relationships of the other mutations are unclear.

In this study we identified 37 newborns with peripheral GALE deficiency. Molecular analysis of the GALE gene in patients with severe defects in GALE activity identified nine mutations including eight missense and one nonsense. 


\section{MATERIALS AND METHODS}

\section{Patients information}

The research was approved by the Seoul National University Bundang Hospital institutional review board and written informed consents were obtained from the subjects and/or their parents. Korean patients with galactosemia were selected from the neonatal screening results for the past few years. They were consulted to Seoul National University Hospital for the enzyme assays of GALK, GALT, and GALE. Measurement of three enzyme activities was carried out in red blood cells by radiometric assay as described elsewhere. ${ }^{14}$

\section{DNA amplification and direct sequencing}

Human genomic DNA was isolated from frozen white blood cells using a QIAamp DNA Blood Mini Kit ${ }^{\circledR}$ (Qiagen, Germany) or a LaboPass ${ }^{\mathrm{TM}}$ Blood Mini kit (Cosmogenetech, Seoul, Korea). Polymerase chain reaction was performed on genomic DNA to amplify all coding exons and flanking intronexon junctions of the GALE gene using a set of 10 primer pairs as described elsewhere. ${ }^{3}$ PCR products were purified using a QIAquick PCR Purification Kit ${ }^{\circledR}$ (Qiagen, Germany) or QIAquick Gel Extraction Kit ${ }^{\circledR}$ (Qiagen, Germany), and sequenced using an ABI Prism ${ }^{\circledR}$ BigDye $^{\text {TM }}$ Terminator Cycle Sequencing Ready Reaction Kit v3.1 protocol. Data were analyzed using an ABI3730XL DNA sequencer (Applied Biosystems, USA).

\section{Confirmation of mutations in GALE by PCR-RFLP}

In order to confirm the presence of the mutations identified by sequencing, PCR products were digested with the restriction enzymes detailed below. Primer pairs used were the same as those used for sequencing except that a mismatched reverse primer of exon 2 (reverse primer: 5'-TGACCACAGGCAAGTAGCTA-3') was used to create a new cleavage site. Restriction enzymes used to confirm each mutation are BfaI, BtgI,
Sau96I, TaqI, Hph1, HaeII, NheI (NEB, USA), and HpaII (KOSCO, Korea) as shown in Table 1.

\section{RESULTS}

We identified 37 Korean patients with reduced GALE activity and an increased galactose-1-phosphate level. None of the patients showed obvious clinical symptoms suggesting that all had peripheral type galactosemia. The mean age of patients was 46 days (range: 14 days-11 months). Patients were subdivided into two groups according to their enzyme activities. Seven patients showed severely reduced enzyme activity of $2.82 \pm$ $2.00 \mu \mathrm{mol} /$ hour/gHb (mean $\pm \mathrm{SD}, 0.8-6.2$, Table 2 ), while the other 30 showed moderately reduced enzyme activity of $15.02 \pm 4.55 \mu \mathrm{mol} /$ hour/gHb (mean $\pm \mathrm{SD}, 6.8-25.1$ ) (mean \pm SD and normal range in the control subjects: $29.47 \pm 7.71,19$ 47).

In order to investigate the underlying defects of GALE deficiency, the GALE gene was sequenced in the 7 Korean patients with severe GALE activity attenuation. Nine mutations were identified: 8 missense mutations (p.A25V, p.R40C, p.D69E, p.E165K, p.R169W, p.R239W, p.G302D, and p.R335H) and a single nonsense mutation (p.W336X). Small deletions or insertions were not detected. With the exception of p.R335H, all mutations proved novel. Six patients were compound heterozygotes and one patient had only one mutation; no homozygote was found (Table 2). PCR-RFLP was performed to confirm the above mutations. When amplified with the appropriate primers (described in Materials and Methods), the mutations either generated or destroyed enzyme restriction sites, as shown in Table 1. In order to exclude common polymorphisms for the described mutations, at least 100 alleles from healthy adult individuals without any clinical symptoms of galactosemia were screened for these nine nucleotide substitutions. No mutation was detected in normal individuals, which

Table 1

Restriction enzymes used to confirm the presence of the nine GALE mutations

\begin{tabular}{|c|c|c|c|c|}
\hline \multirow[b]{2}{*}{ Codon Change } & \multirow[b]{2}{*}{ Nucleotide Substitution } & \multirow[b]{2}{*}{ Restriction Enzyme } & \multicolumn{2}{|c|}{ Product size (bp) } \\
\hline & & & Wild-type & Mutation \\
\hline p.A25V & c. $74 \mathrm{C}>\mathrm{T}$ & $B f a \mathrm{I}$ & 181,20 & 201 \\
\hline p.R40C & c. $118 \mathrm{C}>\mathrm{T}$ & BtgI & $106,118,78$ & 196,106 \\
\hline p.D69E & c. $207 \mathrm{C}>\mathrm{A}$ & Sau96I & $146,51,131$ & 146,182 \\
\hline p.E165K & c. $493 \mathrm{G}>\mathrm{A}$ & TaqI & 213,89 & 302 \\
\hline p.R169W & c. $505 \mathrm{C}>\mathrm{T}$ & HpaII & 226,76 & 302 \\
\hline p.R239W & c. $715 \mathrm{C}>\mathrm{T}$ & HpaII & $65,78,80$ & 143,80 \\
\hline p.G302D & c. $905 \mathrm{G}>\mathrm{A}$ & $H p h \mathrm{I}$ & 132,133 & 265 \\
\hline p.R335H & c. $1004 \mathrm{G}>\mathrm{A}$ & HaeII & 119,198 & 317 \\
\hline p.W336X & c. $1007 \mathrm{G}>\mathrm{A}$ & NheI & 317 & 117,200 \\
\hline
\end{tabular}

Numbering according to GenBank Accession \# NT L41668 (cDNA) and \# NP_000394 
Park et al.

Table 2

Mutation analysis in patients with severely reduced GALE activity

\begin{tabular}{llc}
\hline Patients (age $)$ & \multicolumn{1}{c}{ Mutations } & $\begin{array}{c}\text { Enzyme activity in } \\
\text { RBC }(\mu \mathrm{mol} / \mathrm{hr} / \mathrm{gHb})^{\mathrm{a}}\end{array}$ \\
\hline 1 (27 days) & p.G302D / p.R335H & 0.8 \\
2 (45 days $)$ & p. R169W / p.A25V & 1.5 \\
3 (30 days) & p.D69E / p.G302D & 1.7 \\
4 (31 days $)$ & p.D69E / p.E165K & 1.7 \\
5 (23 days $)$ & p.R40C / p.R169W & 2.9 \\
6 (20 days $)$ & p.W336X / not detected & 4.9 \\
7 (24 days $)$ & p.R239W / p.R335H & 6.2
\end{tabular}

${ }^{\mathrm{a}}$ Mean $\pm \mathrm{SD}$ in 7 patients, $2.82 \pm 2.00 \mu \mathrm{mol} / \mathrm{hr} / \mathrm{gHb} ;$ Mean $\pm \mathrm{SD}$ and normal range in the control subjects, $29.47 \pm 7.71,19-47 \mu \mathrm{mol} / \mathrm{hr} / \mathrm{gHb}$

suggests that the reported mutations might be the cause of GALE deficiency.

In order to determine if the above mutations were present in the 30 moderately affected patients, we performed PCR-RFLP. Seventeen of these 30 patients were found to have one of the above mutations, and the remaining 13 did not. The detected mutations were p.G302D (7/17), p.R239W (5/17), p.R169W (3/17), p.D69E (1/17), and p.E165K (1/17). No significant difference was found between the 17 patients with mutations and the 13 without, in terms of average enzyme activity in RBCs $(14.8 \pm 5.3$ vs. $15.4 \pm 3.6 \mu \mathrm{mol} / \mathrm{hour} / \mathrm{gHb}$, mean $\pm \mathrm{SD})$.

We also investigated the frequency of each mutation in 24 patients who had one or two mutant allele(s). Of the 30 mutant alleles detected in Korean patients with GALE deficiency galactosemia, three amino acid substitutions - p.G302D (9/30), p.R239W (6/30), and p.R169W (5/30) - were found to be most frequent and these accounted for $67 \%$ of all mutations. The other 33\% comprised as follows: p.D69E (3/30), p.E165K (2/ 30), p.R335H (2/30), p.A25V (1/30), p.R40C (1/30), and p.W336X (1/30).

Interestingly, two of the most frequently mutated amino acids, p.G302 and p.R239, are located in a region that was previously shown to undergo major conformational change upon substrate binding by x-ray crystallography. ${ }^{15}$ Moreover, p.G302 and p.R239 are highly conserved among species, thus implying their functional importance.

\section{DISCUSSION}

Sequencing analysis of the coding region of GALE gene in 7 Korean patients with severe GALE activity attenuation identified nine mutations, of which eight were novel. None of these mutations were detected among at least 100 normal alleles, thus excluding the possibility that they represent common polymorphisms. Seventeen of the 30 patients with moderate GALE defects were also found to contain one of the above mutations, whereas the remaining 13 did not. However, since only the presence or absence of the above 9 mutations was tested, we cannot exclude the possibility that these 13 patients might have harbored other GALE mutations. The seven se- verely affected patients selected for GALE gene sequencing analysis showed very low GALE activity in red blood cells, with an average of $2.8 \mu \mathrm{mol} / \mathrm{hour} / \mathrm{gHb}$ (normal range, $19-47 \mu \mathrm{mol} /$ hour/gHb). Sequence analysis showed that 6 of the 7 were compound heterozygotes and that the seventh patient had only one mutation, namely, an amino acid substitution of tryptophan to a stop codon in exon 11 (p.W336X). The patient with a nonsense mutation also showed very low GALE enzyme activity $(4.9 \mu \mathrm{mol} / \mathrm{hour} / \mathrm{gHb})$, which suggested that the second allele might also have been defective. It is possible that a large deletion or mutations in the promoter region existed that was not detected by our sequencing analysis. Alternatively, the truncated form of GALE protein may have acted in a dominant negative manner. Seventeen patients with mild GALE activity defects had heterozygous mutations in GALE gene i.e., p.G302D, p.R239W, p.R169W, p.D69E, and p.E165K. Since GALE activities in these patients were 50\% (14.8 $\pm 5.3 \mu \mathrm{mol} /$ hour/gHb) of normal, it is likely that these mutants were nonfunctional and did not act as dominant negatives.

The structure of human epimerase, as determined by $\mathrm{x}$-ray diffraction analyses suggests that the polypeptide folds into two distinct structural motifs: an N-terminal or nucleotide binding motif, and a C-terminal region responsible for binding the UDP-sugar substrate. ${ }^{16}$ The active site is positioned within the crevice between these two domains. The major conformational changes are limited to the regions defined by p.199Glu to p.240Asp and p.274Gly to p.308Tyr. ${ }^{15}$ Interestingly, the two most common mutations found in our Korean patients, p.R239W and p.G302D, are located in this region. Moreover, these two residues are highly conserved among species, suggesting the importance of these amino acids in structure and/or function of the enyzme. Other than p.R239W and p.G302D, none of the other mutations identified in the present study seems to be involved in substrate binding or to play a decisive role with respect to the active site. However, it is possible that changes in hydrophobicity (p.R169W), electrical charge (p.R40C, p.E165K and p.R169W), or size (p.A25V and p.D69E, and p.R335H) might cause conformational changes that affect enzyme activity. Structural analysis of the mutant proteins is required to explore these possibilities.

The functional consequences of the GALE proteins with selected mutations have been reported in terms of their enzyme activities, protein stabilities, and the phenotypic complementations using a yeast expression studies. ${ }^{7,11,17}$ When expressed in a yeast null background, p.N34S, p.G90E, p.V94M, and p.L183P severely affected GALE activity suggesting the importance of these highly conserved amino acid residues. However, the recent functional studies of p.K257R and p.G319E revealed that both substitutions may be polymorpshism that exist in linkage disequilibrium with other, as yet unidentified causal mutations. ${ }^{17}$ Therefore, in order to determine the functional consequences of the newly identified substitutions in the present study, biochemical studies are mandatory and now under progress. 


\section{ACKNOWLEDGMENTS}

This work was supported by grant from the Seoul National University College of Medicine Research Fund 2003-2005.

\section{References}

1. Gitzelmann R, Steinmann B, Mitchell B, Haigis E. Uridine diphosphate galactose $4^{\prime}$-epimerase deficiency. IV. Report of eight cases in three families. Helv Paediatr Acta 1977;31:441-452.

2. Misumi H, Wada H, Kawakami M, Ninomiya H, et al. Detection of UDP-galactose4-epimerase deficiency in a galactosemia screening program. Clin Chim Acta 1981; 116:101-105.

3. Maceratesi P, Daude N, Dallapiccola B, Novelli G, et al. Human UDP-galactose-4epimerase (GALE) gene and identification of five missence mutations in patients with epimerase-deficiency galactosemia. Mol Genet Metab 1998;63:26-30.

4. Holton JB, Gillett MG, MacFaul R, Young R. Galactosaemia: a new severe variant due to uridine diphosphate galactose-4-epimerase deficiency. Arch Dis Child 1981; 56:885-887.

5. Sardharwalla IB, Wraith JE, Bridge C, Fowler B, et al A patient with severe type of epimerase deficiency galactosaemia. J Inherit Metab Dis 1988;11:249-251.

6. Walter JH, Roberts RE, Besley GT, Wraith JE, et al. Generalized uridine diphosphate-galactose-4-epimerase deficiency. Arch Dis Child 1999;80:374-376.

7. Wohlers TM, Chrostacos NC, Harreman MT, Fridovich-Keil JL. Identification and characterization of a mutation, in the human UDP-galactose-4-epimerase gene, associated with generalized epimerase deficiency galactosemia. Am J Hum Genet 1999;64:462-470.

8. Wohlers TM, Fridovich-Keil JL. Studies of the V94M-substituted human UDPgalactose-4-epimerase enzyme associated with generalized epimerase-deficiency galactosemia. J Inherit Metab Dis 2000;23:713-729.
9. Alano A, Almashanu S, Chinsky JM, Costeas P, et al. Molecular characterization of a unique patient with epimerase-deficiency galactosemia. J Inherit Metab Dis 1998; 21:341-350.

10. Daude N, Gallaher TK, Zeschnigk M, Starzinski-powitz A, et al. Molecular cloning, characterization, and mapping of a full-length cDNA encoding human UDP-galactose-4'-epimerase. Biochem Mol Med 1995;56:1-7.

11. Quimby BB, Alano A, Almashanu S, Desandro AM, Characterization of two mutations associated with epimerase-deficiency galactosemia, by use of a yeast expression system for human UDP-galactose-4-epimerase. Am J Hum Genet 1997;61:590-598.

12. Henderson JM, Huguenin SM, Cowan TM, Fridovich-Keil JL. A PCR-based method for detecting known mutations in the human UDP galactose-4' -epimerase gene associated with epimerase-deficiency galactosemia. Clin Genet 2001; 60:350-355.

13. Thoden JB, Wohlers TM, Fridovich-Keil JL, Holden HM. Molecular basis for severe epimerase deficiency galactosemia. X-ray structure of the human V94Msubstituted UDP-galactose-4-epimerase. J Biol Chem 2001;276:20617-20623.

14. Shin YS. Galactose metabolites and disorders of galactose metabolism. In: Hommes FA, editor. Techniques in diagnostic human biochemical genetics. New York: Wiley-Liss, Inc. 1991:267-283.

15. Thoden JB, Wohlers TM, Fridovich-Keil JL, Holden HM. Crystallographic evidence for Tyr 157 functioning as the active site base in human UDP-galactose-4-epimerase. Biochemistry 2000;39:5691-5701.

16. Thoden JB, Frey PA, Holden HA. Molecular structure of the NADH/UDP-glucose abortive complex of UDP-galactose 4-epimerase from Escherichia coli: Implications for the catalytic mechanism. Biochemistry 1996;35:5137-5144.

17. Wasilenko J, Lucas ME, Thoden JB, Holden HM, et al Functional characterization of the K257R and G319E-hGALE alleles found in patients with ostensibly peripheral epimerase deficiency galactosemia. Mol Genet Metab 2005;84:32-38. 